\title{
Facilely Constructed Two-Sided Microstructure Interfaces Between Electrodes and Cellulose Paper Active Layer: Eco-Friendly, Low-Cost and High- Performance Piezoresistive Sensor
}

\section{Zaihua Duan}

University of Electronic Science and Technology of China

Yadong Jiang

University of Electronic Science and Technology of China

Qi Huang

University of Electronic Science and Technology of China

Si Wang

University of Electronic Science and Technology of China

Qiuni Zhao

University of Electronic Science and Technology of China

Yajie Zhang

University of Electronic Science and Technology of China

Bohao Liu

University of Electronic Science and Technology of China

Zhen Yuan

University of Electronic Science and Technology of China

Yang Wang

University of Electronic Science and Technology of China

Huiling Tai ( $\nabla$ taitai1980@uestc.edu.cn )

University of Electronic Science and Technology of China https://orcid.org/0000-0001-5966-3843

\section{Research Article}

Keywords: Microstructure interface, Polyester conductive tape, Cellulose paper, Carbon ink, Piezoresistive sensor, Wearable applications

Posted Date: March 1st, 2021

DOl: https://doi.org/10.21203/rs.3.rs-235867/v1 
License: (c) (i) This work is licensed under a Creative Commons Attribution 4.0 International License. Read Full License 


\section{Abstract}

The microstructure plays an important role in improving the sensing performance of pressure sensor. However, the design of microstructural active layer of pressure sensor usually involves complex process and expensive raw materials. Herein, the common polyester conductive electrodes and cellulose paper that both have inherent microstructure surface are ingeniously combined to form two-sided microstructure interfaces for low-cost, eco-friendly and high-performance flexible piezoresistive pressure sensor. In order to obtain conductive and low-cost active layer paper, daily carbon ink, which is usually used for writing, is preferred as a conductive material. Meanwhile, we experimentally confirm that the proposed structure is also suitable for other conductive materials, such as carbon nanotubes. The results show that as-fabricated piezoresistive sensor has high pressure sensitivities of 5.54 and $1.61 \mathrm{kPa}^{-1}$ in the wide linear ranges of $0.5-5$ and 5-60 kPa, respectively, and good durability (5000 cycles under $2 \mathrm{kPa}$ ). The sensing mechanism of the piezoresistive sensor is analyzed by combining the characterization results and finite element simulation. Benefitting from the high sensing performance, the good flexibility and non-toxic property, the piezoresistive sensor is demonstrated for multiple wearable applications (e.g., wrist pulse, speech recognition, finger bending, abdominal respiration, counting steps, and pressure distribution). This work provides a simple and universal strategy for the design of piezoresistive sensor from the microstructure interfaces between electrodes and active layer.

\section{Introduction}

With the rapid development of wearable electronics, the flexible pressure sensors are playing an increasingly integral role (Chen et al. 2020; He et al. 2021; Ruth et al. 2020; Zhang et al. 2019; Zheng et al. 2020a). According to different transduction mechanisms, the types of pressure sensors mainly include resistance, capacitance and piezoelectricity. Among them, the resistance type pressure sensor (piezoresistive sensor) is favored by researchers because of its simple read-out signal, facile fabrication technique and high linearity (Zheng et al. 2020a).

In the process of realizing high-performance flexible piezoresistive sensors, the microstructure active layer plays an important role (He et al. 2021; Ruth et al. 2020). A variety of artificial microstructure active layers (such as micropyramid arrays (Choong et al. 2014; Gu et al. 2017; He et al. 2020; Li et al. 2020a; Ma et al. 2020), microdome/hemisphere arrays (Park et al. 2014; Park et al. 2015), hollow-sphere microstructure (Pan et al. 2014), binary spiky/spherical microstructures (Kim et al. 2020), pollen-shaped hierarchical structureand (Zhao et al. 2020b ) have been reported for fabricating flexible piezoresistive sensors. Taking the widely used micropyramid arrays as an example, they usually involve complex processes (such as casting, etching and photolithography), and various raw materials and reagents (Choong et al. 2014; Gu et al. 2017; He et al. 2020; Li et al. 2020a; Ma et al. 2020). For this reason, some materials with inherent microstructure have attracted the attention of researchers. Cellulose paper and fiber fabric have good flexibility, low cost and inherent rough surface structure, which make them excellent materials for flexible piezoresistive sensors (Chen et al. 2021; Ding et al. 2020; Gao et al. 2019; Gong et al. 2014; Guo et al. 2019; Han et al. 2019; Islam et al. 2020; Tai et al. 2020; Tao et al. 2017; Tian et al. 2019; Wu et al. 2020; 
Yang et al. 2019; Zhao et al. 2020a; Zheng et al. 2020b). In particular, many flexible piezoresistive sensors based on stacked cellulose paper active layers have been reported in recent years. For example, Tao et al. developed a piezoresistive sensor using five-layer filter paper as active layer and silver paste as electrodes (Tao et al. 2017). Similarly, Yang et al. proposed a piezoresistive sensor based on eight-layer paper and silver paint electrodes (Yang et al. 2019). Feng's group proposed a piezoresistive sensor based on threelayer airlaid paper and copper electrodes (Han et al. 2019). The multilayer microstructure interfaces formed by stacked paper active layers greatly improve the performance of the piezoresistive sensors, but also increase the fabrication procedures of the sensors (Han et al. 2019; Tao et al. 2017; Yang et al. 2019). Usually, the high performance flexible piezoresistive sensors are constructed by using the interlocked microstructure between the active layers, but the role of electrodes is ignored. To our best knowledge, there is no report of high performance piezoresistive sensor based on a single-layer paper active layer. In addition, the rigid metal electrodes have poor compatibility with the flexible paper, which is not conducive to the flexibility of the piezoresistive sensors (Han et al. 2019; Tao et al. 2017; Yang et al. 2019). Therefore, it is very important to find a suitable flexible electrode material for flexible piezoresistive sensors. In our previous work (Duan et al. 2019b), we have demonstrated that the commercial polyester conductive tape with good conductivity and flexibility can be used to fabricate flexible humidity sensors, endowing it with the potential as the electrode material of flexible piezoresistive sensor. Meanwhile, the polyester conductive tape has rough surface structure, which is expected to form a microstructure interface with the rough paper active layer, promoting the sensing performance of the flexible piezoresistive sensor.

In addition to the microstructure active layer, the conductive active material as a key component of the flexible piezoresistive sensor has also attracted attention. Various conductive active materials, such as poly(3,4-ethylenedioxythiophene-poly(styrenesulfonate) (PEDOT:PSS) (Choong et al. 2014; Yang et al. 2019), carbon nanotubes (CNTs) (Ma et al. 2020; Gu et al. 2017; Park et al. 2014; Zhao et al. 2020b), aluminum-doped zinc oxide (He et al. 2020), reduced graphene oxide (rGO) (Park et al. 2015; Tao et al. 2017; Wu et al. 2020), polypyrrole (PPy) (Ding et al. 2020; Pan et al. 2014; Zhao et al. 2020a), polyaniline (PANI) (Kim et al. 2020), carbon black (CB) (Han et al. 2019), gold nanowires (Gong et al. 2014), silver (Gao et al. 2019; $\mathrm{Tian}$ et al. 2019), $\mathrm{Ti}_{3} \mathrm{C}_{2} \mathrm{~T}_{x}$ (Guo et al. 2019; Zheng et al. 2020b), rGO-CB (Cao et al. 2020; Liu et al. 2019), $\mathrm{Ti}_{3} \mathrm{C}_{2} \mathrm{~T}_{x} / \mathrm{rGO}$ (Ma et al. 2018; Zhu et al. 2019), and graphene (Cao et al. 2021; Cheng et al. 2020), have been used for fabricating piezoresistive sensors. Although these materials have many unique merits for piezoresistive sensors, there are still some disadvantages, such as the inconclusive toxicity of CNTs, the relatively high cost, and the complex preparation process (Duan et al. 2019a; Wang et al. 2019). Even though the CB has a relatively low cost, it still needs high cost and a series of processing procedures to obtain uniform carbon black solution (colloid) that can be used to fabricate piezoresistive sensors (Han et al. 2019; Liu et al. 2019). By paying attention to daily life, we can find mass-produced, green and low-cost conductive materials. Carbon ink, as a material for writing and painting, has the advantages of good stability, low price (about $0.5 \mathrm{USD}$ for $50 \mathrm{~mL}$ ) and environmental protection. In our previous work, we have demonstrated that the conductive property of the carbon ink can 
be used to fabricate low-cost tensile strain sensor (Duan et al. 2019a). Therefore, we speculate that the daily carbon ink has the potential to fabricate low-cost and eco-friendly piezoresistive sensors.

In this work, we focus on fabricating low-cost, eco-friendly and flexible piezoresistive sensor using simple method. For this purpose, the daily polyester conductive tape, cellulose paper and carbon ink are selected as raw materials, and the facile two-sided microstructure interfaces between polyester conductive tape electrodes and single-layer paper active layer are constructed for fabricating flexible piezoresistive sensor. The pressure sensing properties of the piezoresistive sensor are investigated, and its sensing mechanism is analyzed by combining characterizations and finite element simulation. Finally, the proposed flexible piezoresistive sensor is demonstrated to have a bright prospect in multiple wearable applications (e.g., wrist pulse, speech recognition, finger bending, abdominal respiration, counting steps, and pressure distribution).

\section{Experiment Details}

\section{Materials}

Common A4 cellulose paper (80 $\mathrm{g} \mathrm{m}^{-2}$, thickness: $107 \mathrm{~mm}$; Asia Symbol (Guangdong) Paper Co., Ltd, China; about 1 USD for 100 sheets), carbon ink (main components: carbon nanoparticles, deionized water, surfactants, and anti-sedimentation agents; Guizhou Boss Chemical Industry Co., Ltd., China; about 0.5 USD for $50 \mathrm{~mL}$ ), polyester conductive tape (width: $10 \mathrm{~mm}$, thickness: $\sim 130 \mathrm{~mm}$; Shenzhen Huijia Adhesive Products Co., Ltd., China; about 2 USD for $50 \mathrm{~m}$ ) and PI adhesive tape (width: $10 \mathrm{~mm}$, thickness: $\sim 55 \mu \mathrm{m}$, Shenzhen Huijia Adhesive Products Co., Ltd., China; about 0.5 USD for $30 \mathrm{~m}$ ) available in the market were utilized as raw materials for fabricating pressure sensors. The CNTs solution (10 wt\%, Chengdu Organic Chemicals Co., Ltd., China; about 20 USD for $50 \mathrm{~mL}$ ) was also utilized for comparison.

\section{Fabrication of the pressure sensors}

Firstly, the paper was soaked in carbon ink to make the carbon ink fully infiltrate it. Secondly, the carbon ink paper was dried at $60^{\circ} \mathrm{C}$ for $1 \mathrm{~h}$ to remove the solvent of carbon ink. Thirdly, the dried carbon ink paper was cut into a square with an area of $1 \mathrm{~cm}^{2}$. Fourth, the two polyester conductive tapes (the side without adhesive) were combined with the upper and lower surfaces of the carbon ink paper. Finally, the flexible piezoresistive sensor with two-sided microstructure interfaces (S2) was encapsulated with PI tape. For comparisons, the piezoresistive sensors with single-sided microstructure interface (S1) and without microstructure interface (S0) were fabricated by the same process. According to the price of raw materials, the cost of the piezoresistive sensor is extremely low (far less than 0.01 USD for each sensor). In addition, the flexible piezoresistive sensor based on CNTs conductive material was also fabricated using the same method.

\section{Characterization and testing}


The morphologies of materials and piezoresistive sensors were characterized by the transmission electron microscopy (TEM, JEOL JEM-2100F, $200 \mathrm{kV}$ ) and field-emission scanning electron microscopy (SEM, FEI Inspect F, $20 \mathrm{kV}$ ), respectively. $X$-ray diffraction (XRD) patterns and zeta potential of the carbon ink were recorded by Bruker D8 ADVANCE diffractometer (Cu Ka1 radiation, $\lambda=1.5406 \AA$ ) and Zetasizer Nano ZS90 equipment (Malvern Instruments, UK). The 3D profiles of the carbon ink paper and polyester conductive tape were characterized by a laser scanning confocal microscope (Optelics C130, Lasertec Corp., Japan). The pressure sensing properties of the piezoresistive sensor were measured by a homemade pressure testing platform, which was composed of pressure testing machine (SJS-500V, Wenzhou SUNDOO Instruments Co., Ltd., China) and testing instrument (Keithley 4200-SCS) (Pan et al. 2020). The application demonstrations of the piezoresistive sensor were completed on a healthy adult. A constant voltage of $1 \mathrm{~V}$ was loaded on the piezoresistive sensor to obtain the real-time current signal.

\section{Finite element analysis simulation}

The stress distributions between the active layer and electrodes under different applied pressures were conducted via finite element analysis simulation. The random profile models between active layer and electrodes were built by AutoCAD 2014. The maximum bulge heights of the carbon ink paper active layer and polyester conductive tape electrodes were set at 20 and $50 \mathrm{~mm}$, respectively, and the cell structure is based on triangle. The length $(\mathrm{L})$ of the contact interface between electrodes and active layer is $400 \mathrm{~mm}$. In the process of simulation, the lower boundary of the model is fixed and different pressures are applied on the upper boundary.

\section{Results And Discussion}

\section{Characterization of the materials}

Fig. 1a shows the conventional use of the carbon ink and paper for writing. In this study, we will investigate their novel application in piezoresistive sensor. Fig. 1b shows the fabrication procedures and photographs of the piezoresistive sensor with two-sided microstructure interfaces. It should be noted that there is a layer of adhesive on the bottom surface of the polyester conductive tape, but the thin layer of adhesive has no effect on its conductivity (Fig. S1) (Duan et al. 2019b). In order to form a piezoresistive sensor with microstructure interfaces, one side of polyester conductive tape without adhesive should be combined with the carbon ink paper. Therefore, a single-layer paper active layer and two polyester conductive tape electrodes can form a piezoresistive sensor with two-sided microstructure interfaces (named S2).

Fig. 2a shows the TEM image of the carbon ink, indicating that the carbon ink contains solid nanoparticles with the diameters of $20-40 \mathrm{~nm}$. The broad diffraction peak $\left(2 \mathrm{q}=10-35^{\circ}\right)$ of XRD pattern confirms that the solid nanoparticles in carbon ink are amorphous carbon (Fig. S2) (Okamura et al. 2006). The zeta potential of the carbon ink is about $-51 \mathrm{mV}$ (far less than $-30 \mathrm{mV}$ ), proving that the carbon ink has excellent stability and dispersion (Fig. S3) (Li et al. 2008). This is consistent with daily experience that the carbon ink can be stored for many years without affecting the use. The excellent 
stability and dispersion of the carbon ink is beneficial to form uniform conductive layer on the paper (Han et al. 2019). From the SEM image in Fig. $2 b$, the paper is made up of interlaced microfibers, which form a rough surface. The high magnification SEM image in Fig. $2 \mathrm{~b}$ shows that the paper fiber surface is clean. Fig. $2 c$ is the SEM image of the paper with carbon ink. In micron size, the surface morphology of carbon ink paper is basically the same as that of paper, indicating that the rough surface structure of paper is not changed by the introduction of the carbon ink. Fig. $2 \mathrm{~d}$ shows the SEM image of carbon ink paper with high magnification. It is confirmed that the surface of paper fiber is coated by fine carbon nanoparticles, endowing the paper with the function of the conducting active layer of piezoresistive sensor. Fig. $2 \mathrm{e}$ shows the SEM image of the polyester conductive tape, which is composed of the textile structure with a rough surface. Cross section SEM image of the sensor with two-sided microstructure interfaces is show in Fig. 2f. The upper/bottom surfaces of the carbon ink paper and the polyester conductive tape electrodes form two microstructure contact interfaces (red dotted lines), resulting in the piezoresistive sensing response of the sensor. For comparison, the cross-section SEM image of the sensor (named S1) with single-sided microstructure interface is characterized as shown in Fig. S4. It can be seen that only the bottom surface of the paper active layer and the polyester conductive tape form a microstructure contact interface (red dotted line). Because the adhesive side of the polyester conductive adhesive electrode is combined tightly with the carbon paper active layer, there is no microstructure contact interface between the upper surface of the paper active layer and the polyester conductive adhesive tape electrode.

Fig. 3 shows the 3D profiles of the carbon ink paper and polyester conductive tape, indicating that both carbon ink paper and polyester conductive tape have rough surface. The height distribution ranges of the carbon ink paper and polyester conductive tape are about 40-60 and 100-150 mm, indicating that the relative height distribution range of the polyester conductive tape is about 2.5 times that of carbon ink paper. The rough surface structure of the carbon ink paper and polyester conductive tape can form a rough contact interface, which is beneficial to the sensing response of the piezoresistive sensor.

\section{Pressure sensing performance}

Fig. 4 shows the piezoresistive sensing properties of the sensors. As shown in Fig. 4a, the piezoresistive sensor S2 with two-sided microstructure interface has a good and stable pressure sensing response under a wide pressure range of $0.1-70 \mathrm{kPa}$. The current of the sensor S2 is $0.12 \mathrm{~mA}$ under no loading pressure. In addition, the linear current versus voltage $(I-V)$ curves of the sensor $\mathrm{S} 2$ under different pressures, indicating that the stable ohmic contact is formed between the active layer and the electrodes (Fig. S5) (Guo et al. 2019; He et al. 2020). The sensitivity (S) of the piezoresistive sensor is defined as $\mathrm{S}=$ $\delta\left(\Delta I / I_{0}\right) / \delta P$, where $\Delta /$ is the relative change of current $\left(\Delta l=I-I_{0}\right), I$ and $I_{0}$ are the current of the sensor with and without loading pressure, and $P$ is the loading pressure (Han et al. 2019). The responses $\left(\Delta I / I_{0}\right)$ of three sensors (S0 without microstructure interface, S1 and S2) under the different pressures are shown in Fig. 4b. According to the linear fitting results, the sensitivities of the sensor $\mathrm{S} 2$ can divided into two regions: Low-pressure range $\left(0.1-0.5 \mathrm{kPa}, \mathrm{S} 2(1)=5.54 \mathrm{kPa}^{-1}\right)$ and high-pressure range (5-60 kPa, S2(2) $\left.=1.61 \mathrm{kPa}^{-1}\right)$. For comparisons, the piezoresistive sensing properties of the sensor S1 and S0 were also 
investigated. Although the sensor S1 with single-sided microstructure interface also has a good piezoresistive sensing response (Fig. S6), its sensitivities are far less than that of sensor S2. According to the calculation, the sensitivities of sensor $\mathrm{S} 2$ are $5(0.1-0.5 \mathrm{kPa})$ and 6 times (5-60 kPa) that of sensor $\mathrm{S} 1$, respectively. The sensor $\mathrm{S} 0$ has almost no piezoresistive sensing response because there is no microstructure interface between the active layer and the electrodes. In order to obtain the stable response and recovery times of the piezoresistive sensor, the response curve of the sensor S2 is tested in a long and stable switching period (5 s) under the pressure of $2 \mathrm{kPa}$ (Fig. 4c) (Park et al. 2014; Zheng et al. 2020b). The response time and recovery times of the sensor S2 are 280 and $285 \mathrm{~ms}$. Fig. $4 \mathrm{~d}$ shows the current response curves of the sensor $\mathrm{S} 2$ under a pressure of $2 \mathrm{kPa}$ for 5000 cycles, proving its outstanding repeatability and stability (full response cycles shown in Fig. S7) (Han et al. 2019; Gong et al. 2014; Zhao et al. 2020a). Compared with many reported microstructure piezoresistive sensors (Table 1), the sensor S2 in this work has a superior performance on the sensitivity. Notably, the piezoresistive sensor based on common paper, polyester conductive tape and carbon ink has lower cost of raw materials and simpler fabrication procedures. On the whole, it is more competitive than many other sensors including paper-based piezoresistive sensors reported previously.

In addition, we confirmed that the proposed structure of the two-sided microstructure interfaces is also suitable for other conductive materials, such as CNTs. Fig. S8 shows the SEM images and pressure sensing performance of the piezoresistive sensor based on CNTs. Compared with carbon ink paper (Fig. 2c), the surface morphology of the CNTs paper is relatively smooth (Fig. S8a, b). This may be due to the high concentration of carbon ink solution, resulting in a large number of CNTs deposited on the paper surface. Due to the good conductivity and the different deposition amount of the CNTs, the current (1.1 $\mathrm{mA})$ of the CNTs sensor is higher than that of the carbon ink sensor S2 (0.12 mA) under no loading pressure (Fig. S8c). The results show that the CNTs sensor still has good piezoresistive response and high sensitivities (0.1-0.5 $\left.\mathrm{kPa}: 3.49 \mathrm{kPa}^{-1} ; 5-60 \mathrm{kPa}: 1.28 \mathrm{kPa}^{-1}\right)$, proving that the proposed structure of the two-sided microstructure interfaces is successful and universal (Fig. S8d). However, the cost of CNTs is much higher than that of common carbon ink. Taking the raw materials purchased in our work as an example, the cost of the CNTs (about 20 USD for $50 \mathrm{~mL}$ ) is 40 times that of common carbon ink (about 0.5 USD for $50 \mathrm{~mL}$ ). In terms of cost and sensing performance, the carbon ink has more advantages than CNTs.

Table 1. Comparison of the sensor in this paper with reported microstructure piezoresistive sensors. 


\begin{tabular}{|c|c|c|c|c|}
\hline Active layer materials & Main methods & $\begin{array}{l}\text { Sensitivity } \\
\left(\mathrm{kPa}^{-1}\right)^{\mathrm{a}}\end{array}$ & $\begin{array}{l}\text { Pressure } \\
\text { Range } \\
(\mathrm{kPa})\end{array}$ & Ref. \\
\hline PEDOT:PSS/PUD & template/casting & 4.88 & $0.37-5.9$ & $\begin{array}{l}\text { Choong et al. } \\
\text { (2014) }\end{array}$ \\
\hline rGO/paper & soaking/annealing & 0.172 & $0-2$ & Tao et al. (2017) \\
\hline PEDOT:PSS/paper & dip-drying & 1.14 & $<60$ & $\begin{array}{l}\text { Yang et al. } \\
(2019)\end{array}$ \\
\hline PPy/tissue paper & dip-drying & 4.8 & $<5.5$ & $\begin{array}{l}\text { Zhao et al. } \\
\text { (2020a) }\end{array}$ \\
\hline Au nanowires/paper & dip-drying & 1.14 & $0-5$ & $\begin{array}{l}\text { Gong et al. } \\
\text { (2014) }\end{array}$ \\
\hline Au nanowires/paper & dip-drying/priting & 1.5 & $0.03-30.2$ & Gao et al. (2019) \\
\hline $\mathrm{Ti}_{3} \mathrm{C}_{2} \mathrm{~T}_{x} /$ tissue paper & coating/priting & 3.81 & $0.982-10$ & Guo et al. (2019) \\
\hline $\mathrm{Ti}_{3} \mathrm{C}_{2} \mathrm{~T}_{x} /$ cotton fabric & dip-drying & 5.3 & $0-1.3$ & $\begin{array}{l}\text { Zheng et al. } \\
(2020 \mathrm{~b})\end{array}$ \\
\hline rGo/cellulose fabric & soak-annealing & 2.77 & $0-0.2$ & Wu et al. (2020) \\
\hline PPy/yarn & polymerization & 0.187 & $0-15$ & Ding et al. (2020) \\
\hline rGO-CB/paper & spraying & 0.0059 & $0-50$ & Liu et al. (2019) \\
\hline rGO-CB/loofah sponge & self-assembly & 0.66 & $0-0.5$ & Cao et al. (2020) \\
\hline CB/fabric & deposition/coating & 0.585 & $0-35$ & Luo et al. (2016) \\
\hline rGO/PDMS & template method & 0.251 & $0-2.6$ & $\begin{array}{l}\text { Pang et al. } \\
\text { (2018) }\end{array}$ \\
\hline rGO/PANI/sponge & $\begin{array}{l}\text { polymerization/freeze } \\
\text { drying }\end{array}$ & 0.152 & $0-3.24$ & Ge et al. (2018) \\
\hline $\begin{array}{l}\text { common carbon } \\
\text { ink/paper }\end{array}$ & soak-drying & 5.54 & $0.5-5$ & this work \\
\hline
\end{tabular}

aSensitivity $=\delta\left(\Delta l / I_{0}\right) / \delta P$ or $\delta\left(\Delta R / R_{0}\right) / \Delta p$, where $\Delta l / \Delta R$ is the relative change in current/resistance, $I_{0} / R_{0}$ is the current/resistance of the sensor without loading pressure, and $\mathrm{P}$ is the applied pressure.

\section{Pressure sensing mechanism}

To elucidate the effects of the microstructure interface with different layers on sensors' performances, the finite element analysis simulation is implemented to simulate the stress distribution between the active layer and electrodes under different applied pressures (Fig. 5) (Shi et al. 2018). From Fig. 5a, b, the stress distribution of sensor S2 with two-sided microstructure interfaces is larger than that of sensor S1. It 
should be noted that only one-sided microstructure contact interface is formed between the bottom electrode and the active layer of the sensor S1. The upper electrode with adhesive of the sensor S1 is combined tightly with the paper active layer, resulting in no microstructure contact interface (Fig. 5a, SEM image of Fig. S4). Taking the stress distribution of the contact interface between the upper electrode and the active layer of sensor S1 and S2 as an example, the stress distribution of sensor S2 is much larger than that of sensor $\mathrm{S} 1$ under an applied pressure of $50 \mathrm{kPa}$ (Fig. 5c). By comparing the average stress, the average stress values of sensor S2 is also larger than that of sensor S1 (Fig. 5d). Therefore, a larger deformation can occur at the interface between the electrodes and the active layer, promoting the response of the piezoresistive sensor S2 (Pang et al. 2018; Park et al. 2014; Shi et al. 2018). In addition, due to the two-sided microstructure interfaces, the current of sensor S2 (0.12 mA, Fig. 4a) is less than that of sensor S1 (0.57 mA, Fig. S6) under no loading pressure, which provides a larger current response space for the piezoresistive response of the sensor. The above simulation analysis and experimental results are consistent with paper-based piezoresistive sensors with multilayer microstructure reported previously, but in this work, a more simple and low-cost method is used to construct a high-performance paper-based piezoresistive sensor.

\section{Demonstration of multiple applications}

Although the paper has no water resistance, the piezoresistive sensor has good waterproof performance by PI tape encapsulation. As shown in Fig. 6a, the piezoresistive sensor placed in water still has effective response to different finger pressing (Movie S1). In addition, the temperature change in the range of 20-50 ${ }^{\circ} \mathrm{C}$ has no effect on the piezoresistive sensor (Fig. S9). Thanks to the good flexibility of the paper and polyester conductive tape electrodes, and the high sensitivity in wide detection range of the piezoresistive sensor, it can be used for a variety of wearable detection. Fig. $6 \mathrm{~b}$ shows wrist pulse response curves of the sensor before and after running. In the relaxed state, the wrist pulse rate is about 74 beats per minute (bpm). After running, the wrist pulse rate increases rapidly to $108 \mathrm{bpm}$, which is consistent with the normal physiological phenomenon (Gong et al. 2014; Tian et al. 2020). In the detail enlarged view (Fig. 6c), two clearly obvious peaks P1 (systolic peak) and P2 (diastolic peak) can be clearly distinguished, which is similar to previous reports (Choong et al. 2014; Li et al. 2020b; Tian et al. 2020). By attaching the sensor to the larynx, it can be used to recognize different words (Fig. 6d). The sensor can be also used to detect different finger bending (Fig. 6e) and abdominal breathing respiratory rate (Fig. 6f). In addition, the sensor is pasted under the insole and can be used to monitor the foot movement (Fig. 6g, Movie S2). Through simple integration, a sensor array ( $3 * 3)$ is fabricated as shown in Fig. 6h. For simulating the pressure distributions, five weights with different masses are placed in different sensor units of the sensor array (Fig. 6h). By testing the response of each sensor unit, the pressure distributions are revealed as shown in Fig. $6 \mathrm{i}$.

In the fabrication process of the piezoresistive sensor, it shows the characteristics of green environmental protection (without expensive raw materials, toxic reagents and special equipment). In order to reduce the environmental pollution caused by electronic devices, it is necessary to dispose the sensors in a low-cost 
and harmless way. Thanks to the flammability of paper and polyester conductive tape, the piezoresistive sensor can be easily ignited and carbonized, indicating another green feature (Fig. 7) (Zhang et al. 2020).

\section{Conclusion}

In summary, a low-cost, eco-friendly flexible piezoresistive sensor was designed and fabricated by utilizing the common cellulose paper, polyester conductive tape and carbon ink. In the fabrication process, the sensor does not involve complex techniques and expensive/toxic raw materials/reagents. In addition, the piezoresistive sensor has very low cost (far less than 0.01 USD for each sensor) and can be disposed of in an eco-friendly way. By constructing a two-sided microstructure interfaces between the electrodes and the active layer, as-fabricated piezoresistive sensor has high pressure sensitivities of 5.54 and $1.61 \mathrm{kPa}^{-1}$ in the wide linear ranges of $0.5-5$ and $5-60 \mathrm{kPa}$, respectively. Benefitting from the high sensing performance in a wide response range, the piezoresistive sensor has multiple wearable applications (e.g., wrist pulse, speech recognition, finger bending, abdominal respiration, counting steps, and pressure distribution). Combined with the results of sensing performance and mechanism analysis, this study demonstrates a simple and universal strategy for the design of piezoresistive sensor from the microstructure interface between electrodes and active layer. Furthermore, we believe that this work will have significant impacts on the fabrication of other electronic devices using daily materials such as carbon ink and flexible polyester conductive tape.

\section{Declarations}

Acknowledgments This work is supported by the National Science Funds for Excellent Young Scholars of China (Grant No. 61822106) and Natural Science Foundation of China (Grant No. U19A2070).

\section{Compliance with ethical standards}

Conflict of interest The authors declare that there is no conflict of interest regarding the publication of this paper.

Publisher's Note Springer Nature remains neutral withregard to jurisdictional claims in published maps and institutional affiliations.

\section{References}

1. Cao M, Fan S, Qiu H, Su D, Li L, Su J (2020) CB Nanoparticles optimized 3D wearable graphene multifunctional piezoresistive sensor framed byloofah sponge. ACS Appl Mater Interfaces 12:36540-36547. https://doi.org/10.1021/acsami.0c09813

2. Cao M, Su J, Fan S, Qiu H, Su D, Li L (2021) Wearable piezoresistive pressure sensors based on 3D graphene. Chem Eng J 406:126777. https://doi.org/10.1016/j.cej.2020.126777 
3. Chen W, Yan X (2020) Progress in achieving high-performance piezoresistive and capacitive flexible pressure sensors: A review. J Mater Sci Technol 43:175-188.

https://doi.org/10.1016/j.jmst.2019.11.010

4. Chen Z, Yan T, Pan Z (2021) Review of flexible strain sensors based on cellulose composites for multi-faceted applications. Cellulose 28:615-645. https://doi.org/10.1007/s10570-020-03543-6

5. Cheng $L$ et al. (2020) A highly sensitive piezoresistive sensor with interlocked graphene microarrays for meticulous monitoring of human motions. J Mater Chem C 8:11525-11531. https://doi.org/10.1039/d0tc02539a

6. Choong CL et al. (2014) Highly stretchable resistive pressure sensors using a conductive elastomeric composite on a micropyramid array. Adv Mater 26:3451-3458.

https://doi.org/10.1002/adma.201305182

7. Ding X et al. (2020) Highly accurate wearable piezoresistive sensors without tension disturbance based on weaved conductive yarn. ACS Appl Mater Interfaces 12:35638-35646. https://doi.org/10.1021/acsami.0c07928

8. Duan Z et al. (2019a) Inspiration from Daily Goods: A low-cost, facilely fabricated, and environmentfriendly strain sensor based on common carbon ink and elastic core-spun yarn. ACS Sustain Chem Eng 7:17474-17481. https://doi.org/10.1021/acssuschemeng.9b04690

9. Duan Z et al. (2019b) Facile, flexible, cost-saving, and environment-friendly paper-based humidity sensor for multifunctional applications. ACS Appl Mater Interfaces 11:21840-21849. https://doi.org/10.1021/acsami.9b05709

10. Gao L, Zhu C, Li L, Zhang C, Liu J, Yu HD, Huang W (2019) All paper-based flexible and wearable piezoresistive pressure sensor. ACS Appl Mater Interfaces 11:25034-25042. https://doi.org/10.1021/acsami.9b07465

11. Ge G, Cai Y, Dong Q, Zhang Y, Shao J, Huang W, Dong X (2018) A flexible pressure sensor based on $\mathrm{rGO} /$ polyaniline wrapped sponge with tunable sensitivity for human motion detection. Nanoscale 10:10033-10040. https://doi.org/10.1039/c8nr02813c

12. Gong $S$ et al. (2014) A wearable and highly sensitive pressure sensor with ultrathin gold nanowires. Nat Commun 5:3132. https://doi.org/10.1038/ncomms4132

13. Gu Y, Wang X, Gu W, Wu Y, Li T, Zhang T (2017) Flexible electronic eardrum. Nano Res 10:2683-2691. https://doi.org/10.1007/s12274-017-1470-1

14. Guo Y, Zhong M, Fang Z, Wan P, Yu G (2019) A wearable transient pressure sensor made with MXene nanosheets for sensitive broad-range human-machine interfacing. Nano lett 19:1143-1150. https://doi.org/10.1021/acs.nanolett.8b04514

15. Han Z et al. (2019) Ultralow-cost, highly sensitive, and flexible pressure sensors based on carbon black and airlaid paper for wearable electronics. ACS Appl Mater Interfaces 11:33370-33379. https://doi.org/10.1021/acsami.9b12929

16. He F, You X, Wang W, Bai T, Xue G, Ye M (2021) Recent progress in flexible microstructural pressure sensors toward human-machine interaction and healthcare applications. Small Methods 2001041. 
https://doi.org/10.1002/smtd.202001041

17. He K et al. (2020) High-performance zero-standby-power-consumption-under-bending pressure sensors for artificial reflex arc. Nano Energy 73:104743.

https://doi.org/10.1016/j.nanoen.2020.104743

18. Islam GMN, Ali A, Collie S (2020) Textile sensors for wearable applications: a comprehensive review. Cellulose 27:6103-6131. https://doi.org/10.1007/s10570-020-03215-5

19. Kim YR et al. (2020) Binary spiky/spherical nanoparticle films with hierarchical micro/nanostructures for high-performance flexible pressure sensors. ACS Appl Mater Interfaces 12:58403-58411. https://doi.org/10.1021/acsami.0c18543

20. Li D, Muller MB, Gilje S, Kaner RB, Wallace GG (2008) Processable aqueous dispersions of graphene nanosheets. Nat Nanotechnol 3:101-105. https://doi.org/10.1038/nnano.2007.451

21. Li G, Chen D, Li C, Liu W, Liu H (2020a) Engineered microstructure derived hierarchical deformation of flexible pressure sensor induces a supersensitive piezoresistive property in broad pressure range. Adv Sci 7:2000154. https://doi.org/10.1002/advs.202000154

22. Li Z et al. (2020b) Gelatin Methacryloyl-based tactile sensors for medical wearables. Adv Funct Mater 30:2003601. https://doi.org/10.1002/adfm.202003601

23. Liu H et al. (2019) A flexible multimodal sensor that detects strain, humidity, temperature, and pressure with carbon black and reduced graphene oxide hierarchical composite on paper. ACS Appl Mater Interfaces 11:40613-40619. https://doi.org/10.1021/acsami.9b13349

24. Luo $\mathrm{N}$ et al. (2016) Flexible piezoresistive sensor patch enabling ultralow power cuffless blood pressure measurement. Adv Funct Mater 26:1178-1187. https://doi.org/10.1002/adfm.201504560

25. Ma C et al. (2020) Robust flexible pressure sensors made from conductive micropyramids for manipulation tasks. ACS Nano 14:12866-12876. https://doi.org/10.1021/acsnano.0c03659

26. Ma Y et al. (2018) 3D synergistical MXene/reduced graphene oxide aerogel for a piezoresistive sensor. ACS Nano 12:3209-3216. https://doi.org/10.1021/acsnano.7b06909

27. Okamura $\mathrm{M}$ et al. (2006) Acid-catalyzed reactions on flexible polycyclic aromatic carbon in amorphous carbon. Chem Mater 18:3039-3045. https://doi.org/10.1021/cm0605623

28. Pan $\mathrm{H}$ et al. (2020) Surface engineering of a 3D topological network for ultrasensitive piezoresistive pressure sensors. ACS Appl Mater Interfaces 12:38805-38812.

https://doi.org/10.1021/acsami.0c11658

29. Pan L et al. (2014) An ultra-sensitive resistive pressure sensor based on hollow-sphere microstructure induced elasticity in conducting polymer film. Nat commun 5:3002. https://doi.org/10.1038/ncomms4002

30. Pang $Y$ et al. (2018) Epidermis microstructure inspired graphene pressure sensor with random distributed spinosum for high sensitivity and large linearity. ACS Nano 12:2346-2354. https://doi.org/10.1021/acsnano.7b07613 
31. Park, J et al. (2014) Giant tunneling piezoresistance of composite elastomers with interlocked microdome arrays for ultrasensitive and multimodal electronic skins. ACS Nano 8:4689-4697. https://doi.org/10.1021/nn500441k

32. Park J, Kim M, Lee Y, Lee HS, Ko H (2015) Fingertip skin-inspired microstructured ferroelectric skins discriminate static/dynamic pressure and temperature stimuli. Sci Adv 1:1500661. https://doi.org/10.1126/sciadv.1500661

33. Ruth SRA, Feig VR, Tran H, Bao Z (2020) Microengineering pressure sensor active layers for improved performance. Adv Funct Mater 30:2003491. https://doi.org/10.1002/adfm.202003491

34. Shi J, Wang L, Dai Z, Zhao L, Du M, Li H, Fang Y (2018) Multiscale hierarchical design of a flexible piezoresistive pressure sensor with high sensitivity and wide linearity range. Small 14:1800819. https://doi.org/10.1002/smll.201800819

35. Tai H, Duan Z, Wang Y, Wang S, Jiang Y (2020) Paper-based sensors for gas, humidity, and strain detections: A review. ACS Appl Mater Interfaces 12:31037-31053. https://doi.org/10.1021/acsami.0c06435

36. Tao LQ et al. (2017) Graphene-paper pressure sensor for detecting human motions. ACS Nano 11:8790-8795. https://doi.org/10.1021/acsnano.7b02826

37. Tian K, Sui G, Yang P, Deng H, Fu Q (2020) Ultrasensitive thin-film pressure sensors with a broad dynamic response range and excellent versatility toward pressure, vibration, bending, and temperature. ACS Appl Mater Interfaces 12:20998-21008. https://doi.org/10.1021/acsami.0c05618

38. Tian M, Lu Y, Qu L, Zhu S, Zhang X, Chen S (2019) A pillow-shaped 3D hierarchical piezoresistive pressure sensor based on conductive silver components-coated fabric and random fibers assembly. Ind Eng Chem Res 58:5737-5742. https://doi.org/10.1021/acs.iecr.9b00035

39. Wang C, Xia K, Wang H, Liang X, Yin Z, Zhang Y (2019) Advanced carbon for flexible and wearable electronics. Adv Mater 31:1801072. https://doi.org/10.1002/adma.201801072

40. Wu R et al. (2020) Graphene decorated carbonized cellulose fabric for physiological signal monitoring and energy harvesting. J. Mater Chem A 8:12665-12673. https://doi.org/10.1039/d0ta02221g

41. Yang T, Mativetsky JM (2019) Paper-based mechanical sensors enabled by folding and stacking. ACS Appl Mater Interfaces 11:26339-26345. https://doi.org/10.1021/acsami.9b06071

42. Zhang $\mathrm{H}$ et al. (2020) Heterogeneously integrated flexible microwave amplifiers on a cellulose nanofibril substrate. Nat Commun 11:3118. https://doi.org/10.1038/s41467-020-16957-4

43. Zhang L, Li H, Lai X, Gao T, Liao X, Chen W, Zeng X (2019) Carbonized cotton fabric-based multilayer piezoresistive pressure sensors. Cellulose 26:5001-5014. https://doi.org/10.1007/s10570-01902432-x

44. Zhao P, Zhang R, Tong Y, Zhao X, Tang Q, Liu Y (2020a) All-paper, all-organic, cuttable, and foldable pressure sensor with tuneable conductivity polypyrrole. Adv Electron Mater 6:1901426. https://doi.org/10.1002/aelm.201901426 
45. Zhao T, Yuan L, Li T, Chen L, Li X, Zhang J (2020b) Pollen-shaped hierarchical structure for pressure sensors with high sensitivity in an ultrabroad linear response range. ACS Appl Mater Interfaces 12:55362-55371. https://doi.org/10.1021/acsami.0c14314

46. Zheng Q, Lee JH, Shen X, Chen X, Kim JK (2020a) Graphene-based wearable piezoresistive physical sensors. Mater Today 36:158-179. https://doi.org/10.1016/j.mattod.2019.12.004

47. Zheng Y et al. (2020b) Conductive MXene/cotton fabric based pressure sensor with both high sensitivity and wide sensing range for human motion detection and E-skin. Chem Eng J 127720. https://doi.org/10.1016/j.cej.2020.127720

48. Zhu M et al. (2019) Hollow MXene sphere/reduced graphene aerogel composites for piezoresistive sensor with ultra-high sensitivity. Adv Electron Mater 6:1901064.

https://doi.org/10.1002/aelm.201901064

\section{Figures}
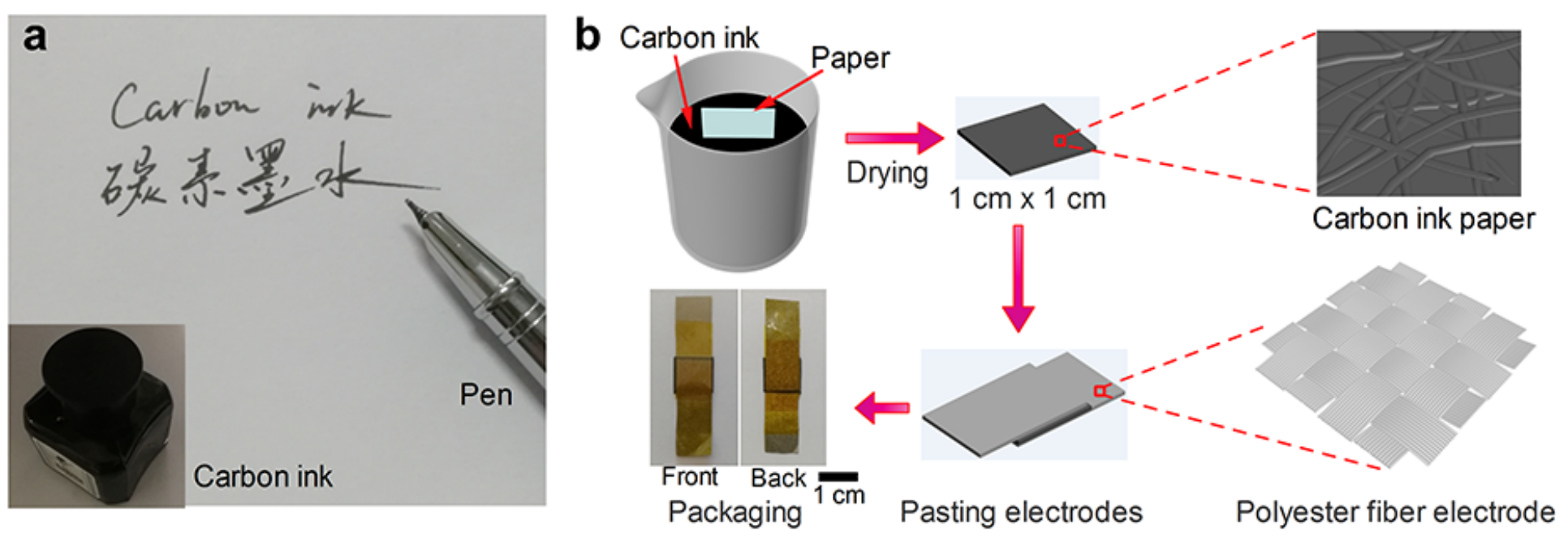

\section{Figure 1}

a Demonstration of the use of carbon ink and paper for writing. b Schematic illustration of fabrication procedures of the piezoresistive sensor with two-sided microstructure interface. 


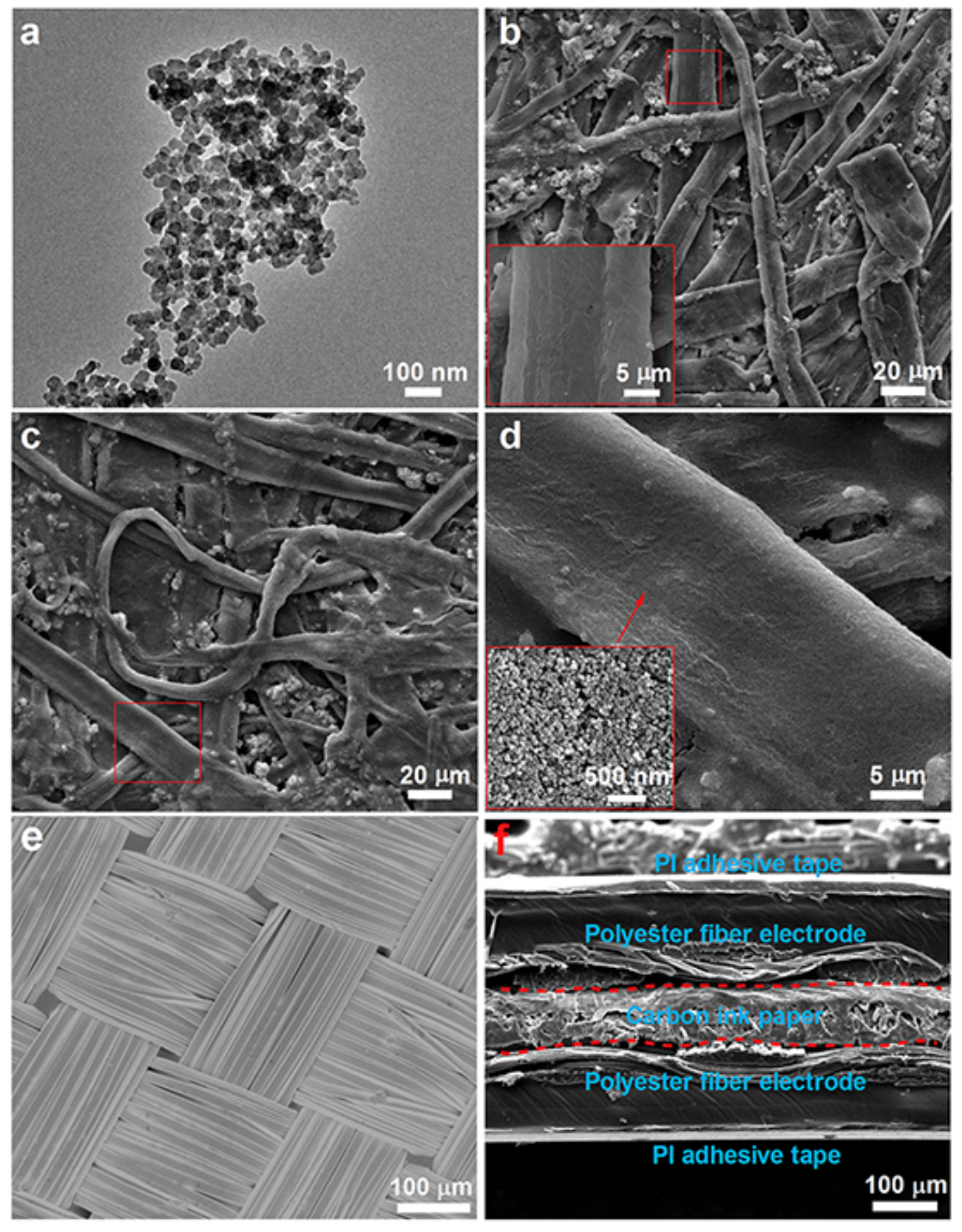

Figure 2

a TEM image of the carbon ink. b SEM image of the paper, and the inset shows the high magnification SEM image. c SEM image of the paper with carbon ink. d High magnification SEM image. e SEM image of the polyester conductive tape. $\mathrm{f}$ Cross-section SEM image of the sensor S2 with two-sided microstructure interfaces. 

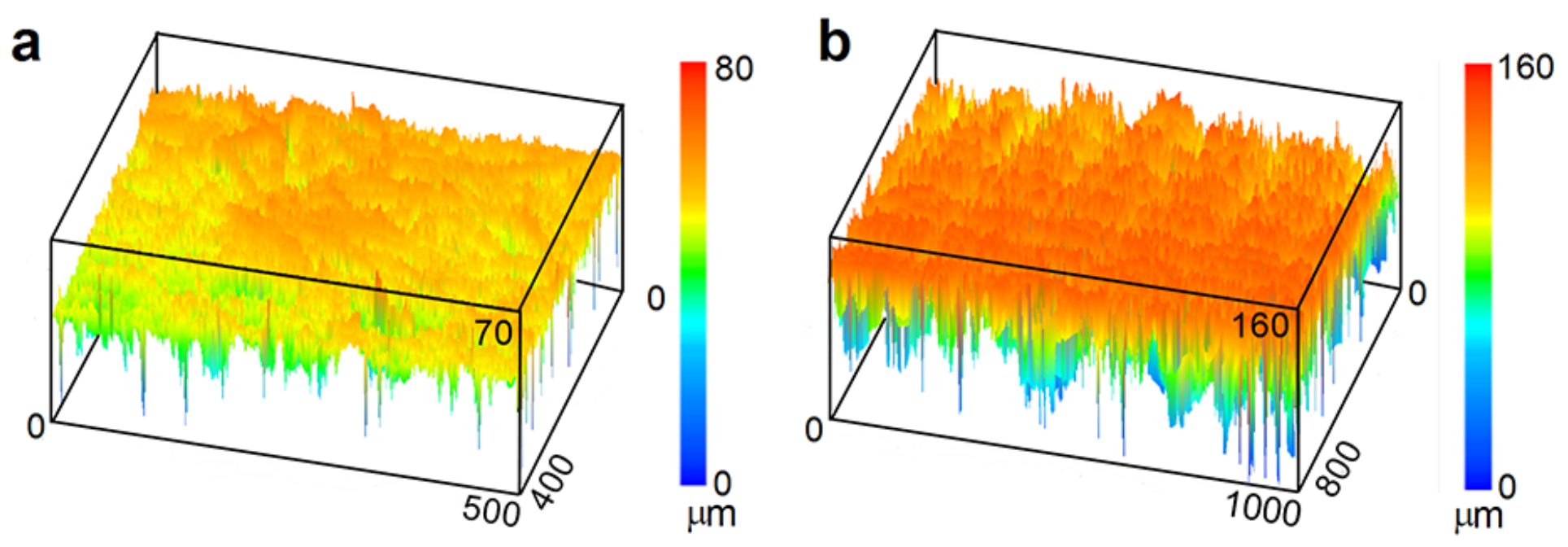

Figure 3

3D profiles profiles of a carbon ink paper, and b polyester conductive tape.
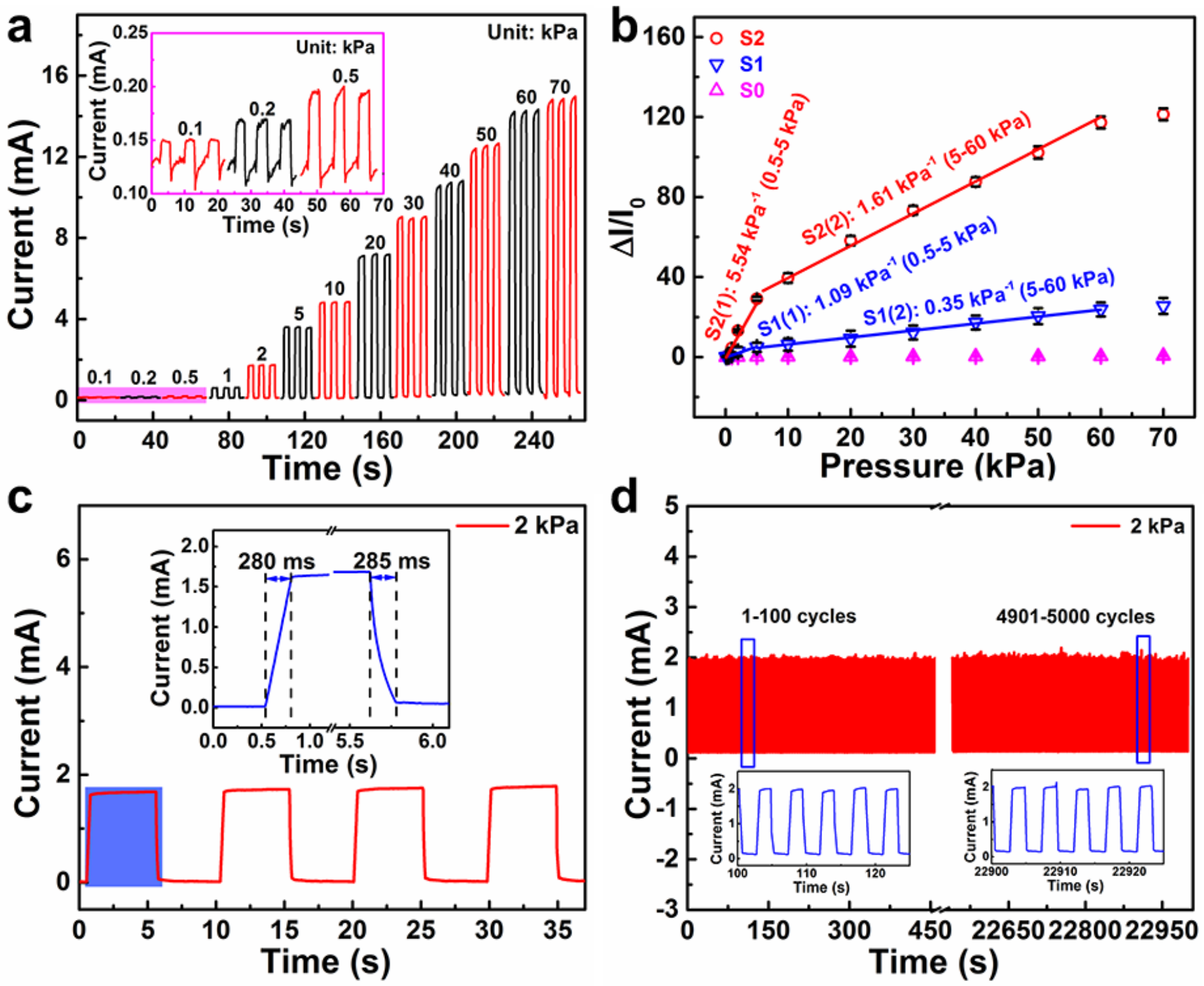
Figure 4

a Current response of the sensor $\mathrm{S} 2$ to increased cyclic pressures $(0.1-70 \mathrm{kPa})$, and the inset shows the current response in the low-pressure range of $0.1-0.5 \mathrm{kPa}$. b Responses of different sensors to different pressures and corresponding linear fitting curves. c Four repetitive response curves of the sensor S2 at 2 $\mathrm{kPa}$, and the inset shows the enlarged current response curve. $\mathrm{d}$ Durability test of the sensor $\mathrm{S} 2$ under a pressure of $2 \mathrm{kPa}$ for 5000 cycles.
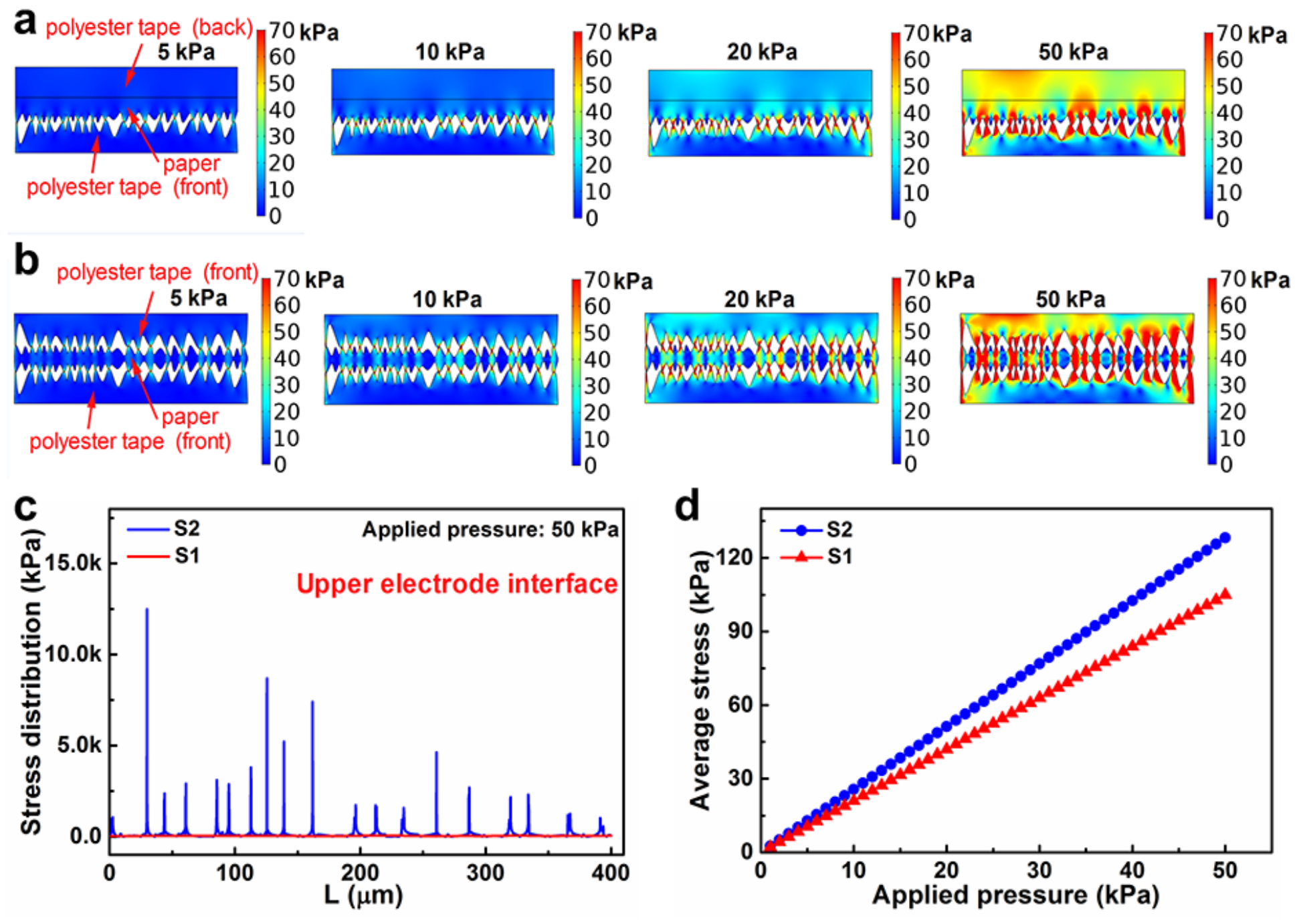

Figure 5

a, b Stress distributions of the contacted microstructure of sensors S1 and sensors S2 with the applied pressure (5-50 kPa). c Stress distributions between the upper electrode and the active layer of the sensor $\mathrm{S} 1$ and S2 under an applied pressure of $50 \mathrm{kPa}$. d Average stress values of the sensor S1 and S2 under different pressures. 

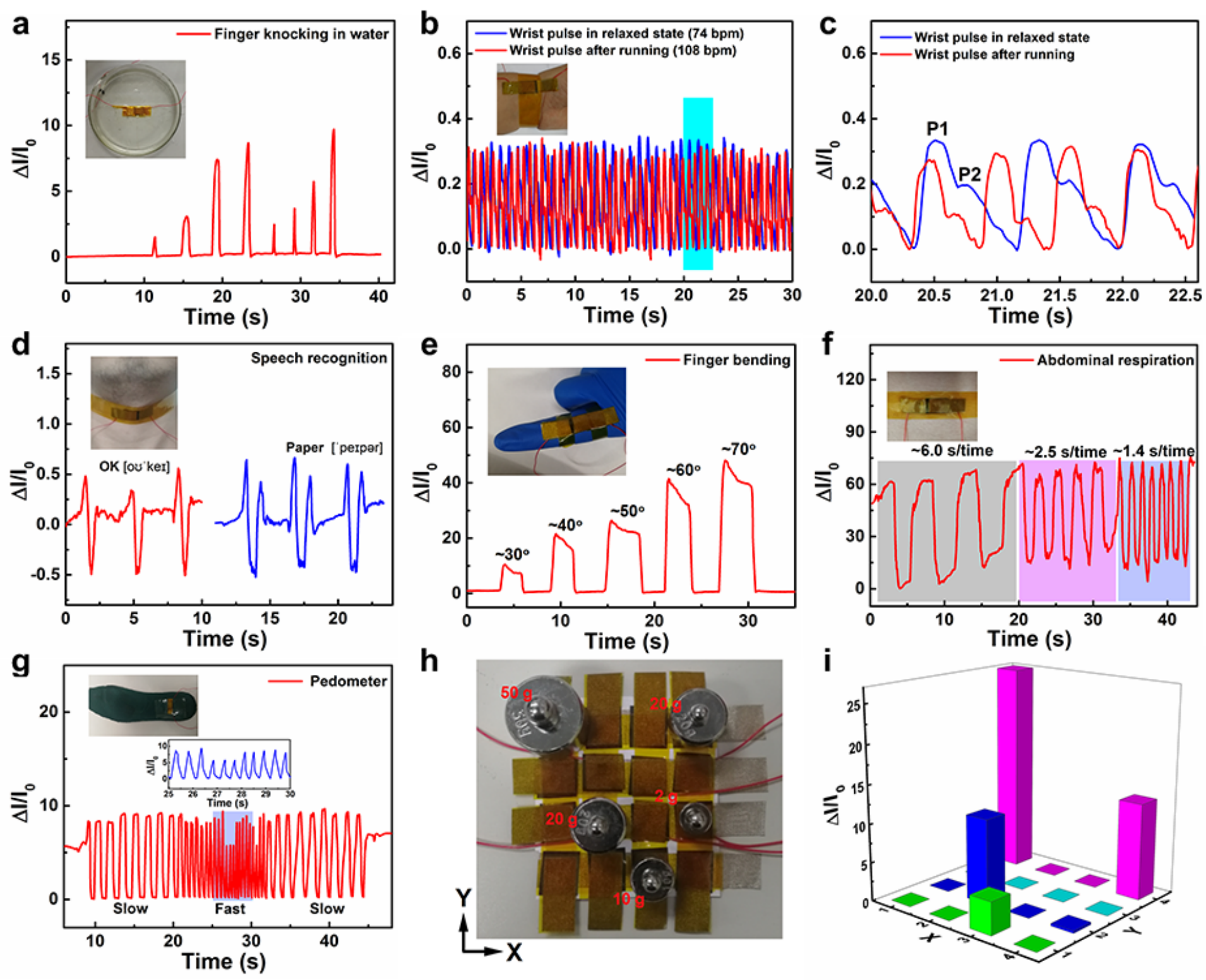

Figure 6

a Current response curve to different finger pressure in water environment. b Wrist pulse response curves before and after running. c Detail enlarged view. $d$ Speech recognition (repeating three times for each pronunciation). e Current response curve to different finger bending state. $f$ Current response curve to different abdominal respiration rates. g Verification of pedometer function. h Photograph of the different weight distribution on sensor array. i Corresponding pressure distribution response. Operation voltage: 1 V. 

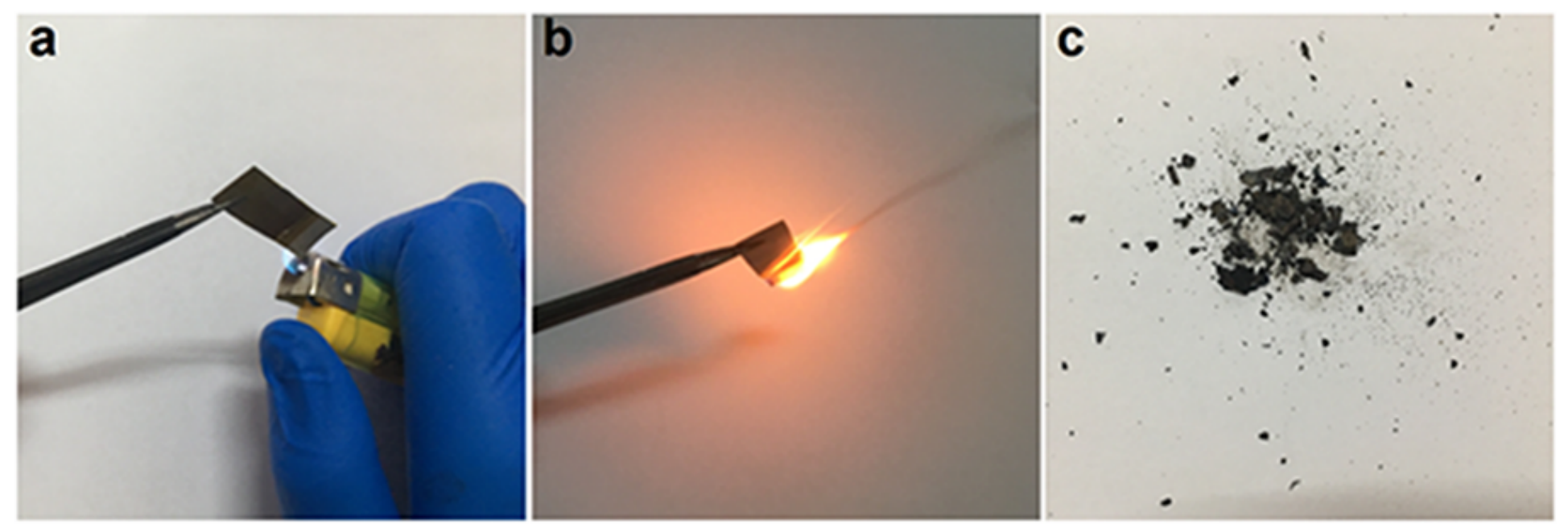

Figure 7

Photographs of disposable and degradable characteristics of the sensor. a ignition, b burning, $\mathrm{c}$ after combustion. The sensor is easily ignited and can be carbonized within about $7 \mathrm{~s}$ in air and then becomes ashes via slightly crushing.

\section{Supplementary Files}

This is a list of supplementary files associated with this preprint. Click to download.

- MovieS1.avi

- MovieS2.avi

- Graphicabstract.tif

- SupplementaryMaterial.docx 